\title{
Parallel, Miniaturized Scanning Probe Microscope for Defect Inspection and Review
}

\author{
H. Sadeghian*ab , T. C. van den Dool ${ }^{\mathrm{a}}$, W. E. Crowcombe ${ }^{\mathrm{a}}$, R. W. Herfst ${ }^{\mathrm{a}}$, J. Winters ${ }^{\mathrm{a}}$, G. F. I. J. \\ Kramer $^{\mathrm{a}}$, N. B. Koster ${ }^{\mathrm{a}}$ \\ ${ }^{a}$ Netherlands Organization for Scientific Applied Research, TNO, Stieltjesweg 1, 2628 CK, Delft,

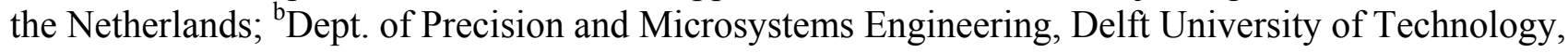 \\ 2628 CD, delft, The Netherlands
}

\begin{abstract}
With the device dimensions moving towards the $1 \mathrm{X}$ node, the semiconductor industry is rapidly approaching the point where $10 \mathrm{~nm}$ defects become critical. Therefore, new methods for improving the yield are emerging, including inspection and review methods with sufficient resolution and throughput. Existing industrial tools cannot anymore fulfill these requirements for upcoming smaller and 3D features, since they are performing at the edge of their performance. Scanning probe microscopy (SPM) has the ability to accurately measure dimensions in the micrometer to nanometer scale. Examples of applications are surface roughness, channel height and width measurement, defect inspection in wafers, masks and flat panel displays. In most of these applications, the target area is very large, and, therefore, the throughput of the measurement plays an important role in the final production cost.

Single SPM has never been able to compete with other inspection systems in terms of measurement speed, thus has not fulfilled the industry needs in throughput and cost. Further increase of the speed of the single SPM helps, but it still is far from the required throughput and, therefore, insufficient for high-volume manufacturing.

Over the past three years, we have developed a revolutionary concept for a multiple miniaturized SPM heads system, which can inspect and measure many sites in parallel. The very high speed of each miniaturized SPM unit allow the user to scan many areas, each with the size of tens of micrometers, in a few seconds. This paper presents an overview of the technical developments and experimental results of the parallel SPM system for wafer and mask inspection.
\end{abstract}

Keywords: Parallel SPM, Miniaturized SPM, Defect inspection, Defect review, Patterned wafer inspection , Mask inspection

\section{INTRODUCTION}

With the shrinking of the device size towards $10 \mathrm{~nm}$ and increasing device complexity, the existing metrology solutions are pushed to their limits of performance. Besides the challenges of device's dimension metrology, detecting and characterizing smaller defects are becoming critical. Moreover, the contrast between defects of interests and nuisance defects becomes so low that it makes the recognition very challenging. In recent years, scanning probe microscopy (SPM) emerged as an option for metrology and inspection. Due to its sub-nanometer imaging and characterization resolution, critical dimensions, channel height and width, and sub-10 $\mathrm{nm}$ defects and particles can be measured and reviewed in nanometer scale. However, single SPM has never been able to compete with other inspection systems in throughput, thus has not fulfilled the industry needs in throughput and cost. Recent developments have significantly pushed the speed of SPM [1,2] and showed the capability of this tool to detect and review defects with the size of sub-10 $\mathrm{nm}$. Howevere, the throughput is still far from its required value and therefore not yet sufficient for metrology and inspection in high-volume manufacturing.

Other attempts to increase the throughput of SPM for large area inspection include parallelization of SPM probes. Multiple probes were fabricated via MEMS fabrications process [3]. In this case, the throughput of SPM is increased in direct proportion to the number of cantilevers in an array. Howevere, there is still a long path to develop a massive parallel SPM to be used in manufacturing environment. Examples are limited number of wires, exchange of one probe in an array, cross talk, data transfer and reliability of the system.

*hamed.sadeghianmarnani@tno.nl

Metrology, Inspection, and Process Control for Microlithography XXVIII, edited by Jason P. Cain, Martha I. Sanchez, Proc. of SPIE Vol. 9050, 90501B · C 2014 SPIE · CCC code: 0277-786X/14/\$18 · doi: 10.1117/12.2045557 
We have recently developed a concept based on 1) increasing the speed of SPM and 2) parallelization of many SPMs to increase the overall throughput so high that it can fulfill the requirements of semiconductor applications [4].

The system enables simultaneous operation of many miniaturized SPM (MSPM) heads on a relatively large sample, such as wafer or mask (Figure 1).

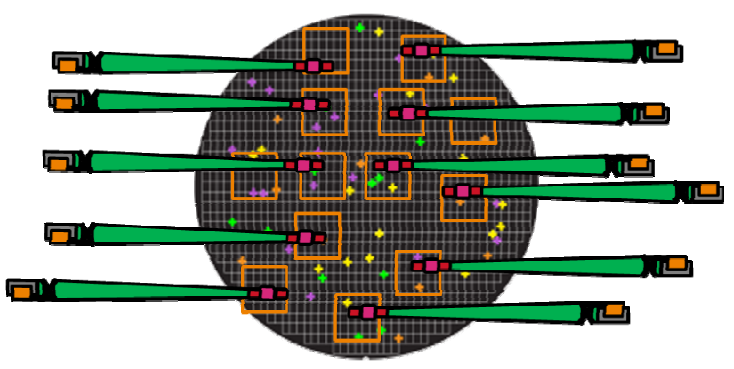

(a)

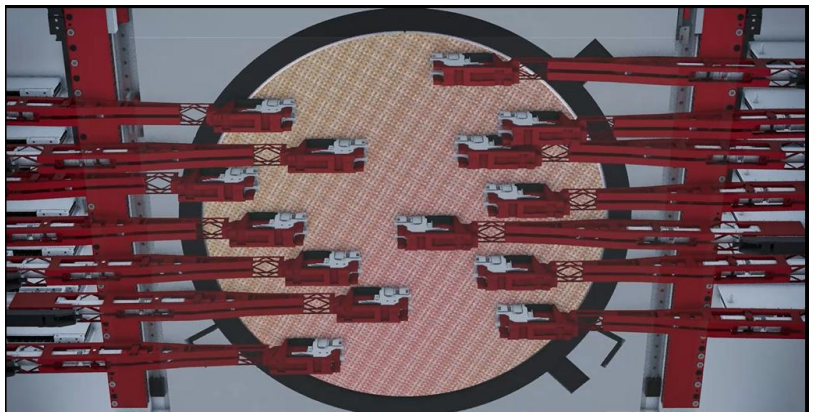

(b)

Figure 1. Overview of parallel SPM. (a) schematic illustration of parallel MSPM to image several locations on a wafer or mask. (b) detail with wafer stage in the middle, up to $2 \times 50$ positioning arms on two sides of the wafer stage, each capable of moving a MSPM scan head on to the $450 \mathrm{~mm}$ wafer. Many parallel MSPM heads enable full wafer coverage at high throughput. Each of the MSPM heads is at the end of a positioning unit that enables moving each MSPM head to a desired site to be scanned. After positioning the MSPM heads are fixed and the wafer stage carries out the scan for all sites simultaneously. Only few arms are shown but the baseline design has $50(2 \times 25)$ MSPM heads on as many arms.

The concept has been designed based on the requirements for defects inspection and review applications, but it can also be implemented for other process control and metrology applications. As it can be seen, the concept consists of two critical sub-systems:

1- High speed parallel positioning unit: This positioning unit consists of parallel mechatronics arms, each capable of positioning a MSPM towards a wafer. Positioning of each MSPM is done individually to enable maximizing the wafer surface coverage. The positioning is done in closed loop to achieve high positioning accuracy. The experimental results revealed a positioning accuracy better than $200 \mathrm{~nm}$.

2- High speed MSPM: A miniature scanning probe microscope (Size $\sim 70 \times 19 \times 45 \mathrm{~mm} 3$ ) has been designed and realized to scan an area of $10 \times 10 \mu \mathrm{m}^{2}$ to detect and review the defects. The scanner has a bandwidth of $\sim 45$ $\mathrm{KHz}$ and a vertical stroke of $2.1 \mu \mathrm{m}$. The read-out of the probe has a noise level of $15 \mathrm{fm} / \sqrt{ } \mathrm{Hz}$ and a bandwidth of more than $3 \mathrm{MHz}$, which allows the use of ultra-high frequency cantilevers. Consequently, a very high speed scanning has been achieved.

To prevent contamination of the wafer with particles, the system will be realized upside-down, that is, with the probes of the SPM heads looking upward to the wafer surface, which is facing downward. By doing so, particles that are generated in the tool will not fall onto the wafer, see Figure 2. Although wafers are placed face up in the Front Opening Unified Pod (FOUP), the required flip of the wafer during loading/unloading is not considered a problem as wafer flipper equipment is commercially available. 


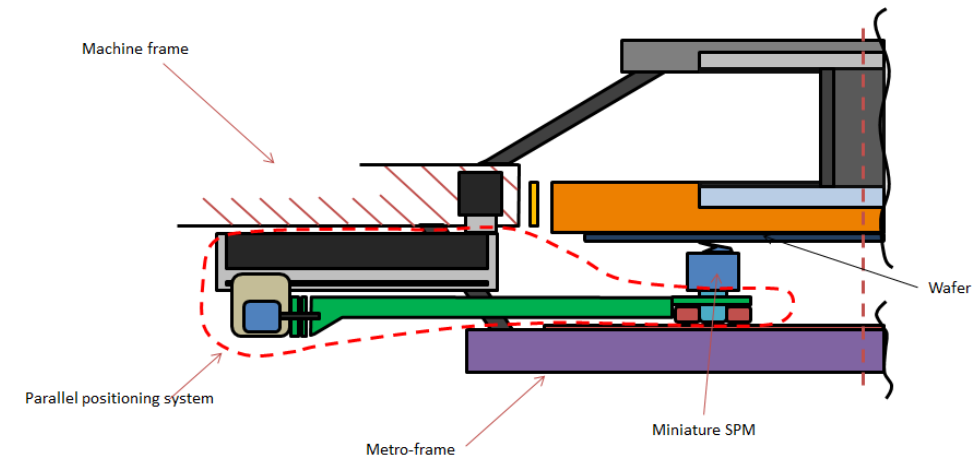

Figure 2:Schematic of the physical architecture of the system. half of the system is illustrated.

In the following sections we present the performance of critical sub-systems, and the progress towards a demonstrator.

\section{PARALLEL POSITIONING UNIT}

The parallel positioning unit performs the following functions; it positions each MSPM head to a pre-defined location $(x$, $y$ axis) on a $450 \mathrm{~mm}$ wafer using a defect location map provided from either a prior wafer inspection tool or defined by the user. It also measures and captures the position ( $x, y$ axis) of each MSPM head. During approach of the wafer and during scanning it keeps the scan heads stationary. Finally it retracts the scan heads from the scanning areas to a home position. The positioning system consists of (see Figure 3) three main actuators for $\mathrm{x}, \mathrm{y}$, and $\mathrm{z}$ positioning of SPM scan heads, a parallel metrology system to measure the location of each scan head, and the mechanical frame, named arm. The positioning system covers the full area of the wafer with up to $50 \mathrm{arms}$ from two sides of the wafer. The long stroke of the arm is about $350 \mathrm{~mm}$ to cover from the edge to the middle of the wafer, and the short stroke of the arm is $\pm 19 \mathrm{~mm}$ in a direction perpendicular to the long stroke.

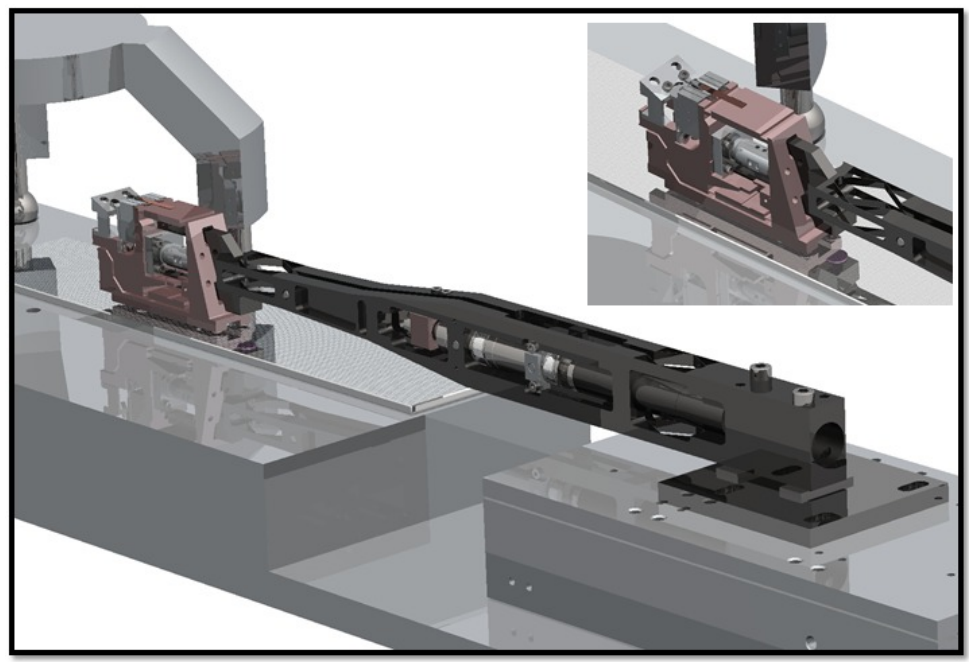

Figure 3:CAD illustration of one positioning arm that carries the MSPM. The inset shows the MSPM.

Figure 3 shows the final design of one positioning arm that carries one MSPM. The experimental results revealed a positioning accuracy better than $200 \mathrm{~nm}$. Figure 4 shows the experimental test setup and the results of positioning repeatability. Figure 5 shows the results from the xy positioning tests with the realized setup from Figure 3. 


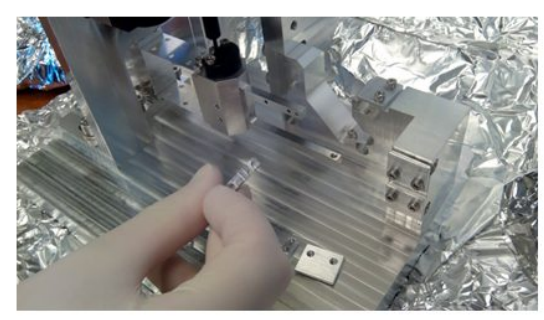

(a)

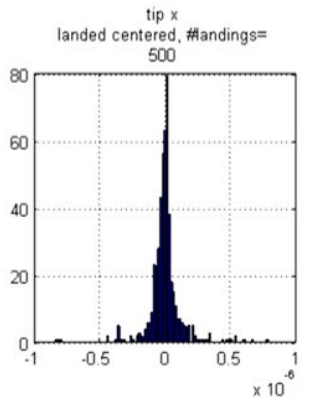

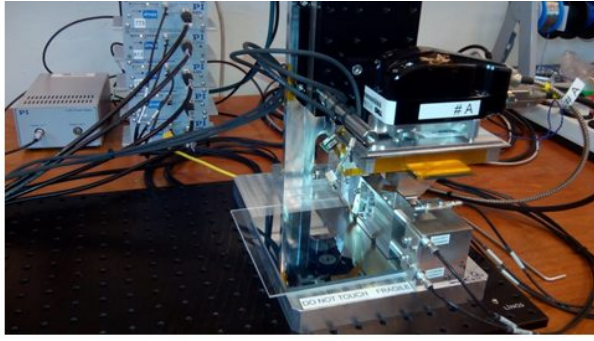

(b)

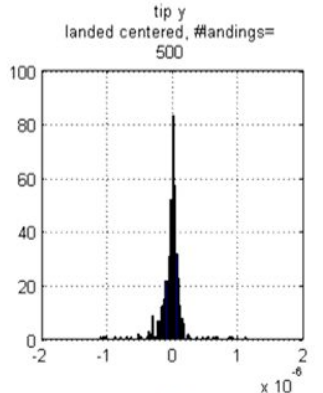

(c)

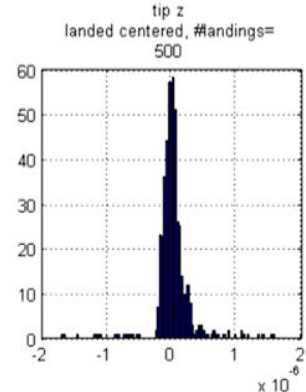

Figure 4: (a) The MSPM positioning repeatability measurement test setup. (b) The setup including 5 capacitive sensors and one interferometer to measure the positioning repeatability in 6 DOF. (c) The experimental results of tip positioning in $\mathrm{x}, \mathrm{y}$ and $\mathrm{z}$ direction, indicating a repeatability of better than $200 \mathrm{~nm}$.
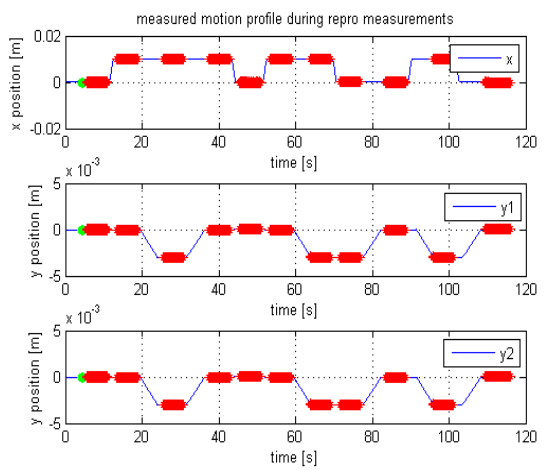

(a)
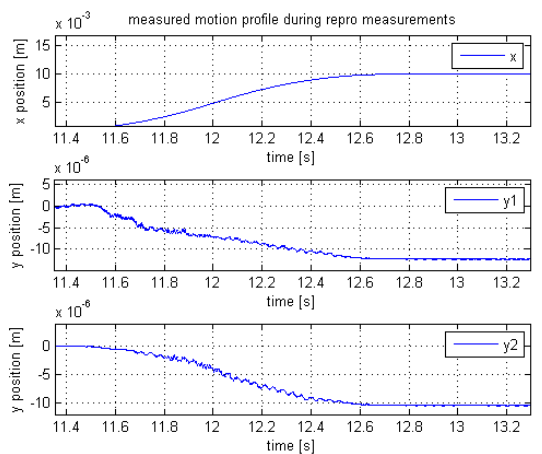

(b)
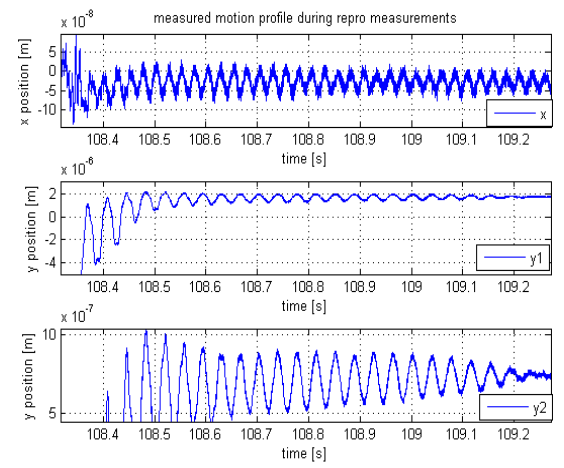

(c)

Figure 5: (a) The xy-motion profile applied to the arm holding the MSPM, measured with the grid encoder under the MSPM. The data from the $\mathrm{x}$ and the two y encoders are shown. (b) The decay of the residual vibrations at the MSPM after an $\mathrm{x}$ positioning step. Some crosstalk from $\mathrm{x}$ to $\mathrm{y}$ and $\mathrm{y}$ to $\mathrm{x}$ is visible indicating the need for this measurement type. 10um crosstalk at $10 \mathrm{~mm}$ step, most likely due to misalignment of the $\mathrm{x}$-stage. (c) The stability of the MSPM on the arm before landing of the device. Less than 10nm in $\mathrm{x}$-direction and less than $100 \mathrm{~nm}$.

\section{MINIATURIZED, HIGH SPEED SPM}

A miniature scanning probe microscope (Size $\sim 70 \times 19 \times 45 \mathrm{~mm}^{3}$ ) has been designed and realized to scan an area of $10 \times$ $10 \mu \mathrm{m}^{2}$ to detect and review the defects. A critical part of this high speed MSPM is the stage that is used to adjust the distance between the sample and the cantilever. This z-scanner has a bandwidth of $45 \mathrm{KHz}$ and a stroke of $2.1 \mu \mathrm{m}$. The 
read-out of the probe (using optical beam deflection) has a resolution of $15 \mathrm{fm} / \sqrt{\mathrm{Hz}}$ and a bandwidth of $3 \mathrm{MHz}$, which allows the use of ultra-high frequency cantilevers. Since a $2.1 \mu \mathrm{m}$ stroke is not sufficient for coarse adjustment of the distance between a sample and the cantilever, an approach stage moves the whole assembly of z-stage and OBD system in the z-direction. A picture of the miniaturized SPM demonstrator is shown in 6, where the z-stage, cantilever holder, approach motor, and the PCB with quadrant cell for the OBD can be seen.

Apart from high scanning speed, a fast approach of the MSPM towards the sample is necessary for high throughput. For this the approach motor in quick succession makes steps of about $1 \mu \mathrm{m}$ while the z-stage is fully extended and a dither piezo drives the cantilever at a resonance frequency. If during a step of the approach motor the amplitude of the cantilever is reduced by a small amount (usually set to $10-25 \%$ ), the z-stage quickly retracts the z-stage and the fast approach is stopped, thereby preventing a crash. The system then switches to a regular approach method where after each step of the stepper-motor the z-stage is scanned from fully retracted until the cantilever is engaged with the sample.

Preliminary experimental results of z-scanner frequency spectra and the noise performance of the optical beam deflection of the MSPM are illustrated in 7. 7a shows that the first resonance of the z-stage is at a frequency of $45 \mathrm{kHz}$. In Figure $7 \mathrm{~b}$ it can be seen that the noise of the OBD is low enough to resolve the thermal movement of a Ultra High Frequency cantilever.

Figure 8 shows images taken with the MSPM illustrating excellent performance on two different samples. In Figure 8a, a $30 \times 30 \mu \mathrm{m}^{2}$ scan of a $3 \mu \mathrm{m}$ pitch grating pattern is shown. It was scanned at 10 lines/s, which is challenging considering the steep $130 \mathrm{~nm}$ steps in the sample and the broad $30 \mu \mathrm{m}$ scan width resulting in a linear velocity of $0.6 \mathrm{~mm} / \mathrm{s}$. Even so, at the moment the scanning speed is limited by the frequency response of the XY-stage; z-stage and feedback still have significant head-room for faster scanning. The result in Figure $8 \mathrm{~b}$ of a $5 \times 5 \mu \mathrm{m}^{2}$ scan of an alignment marker in a resist layer shows that the system can also produce very detailed images of a soft sample.

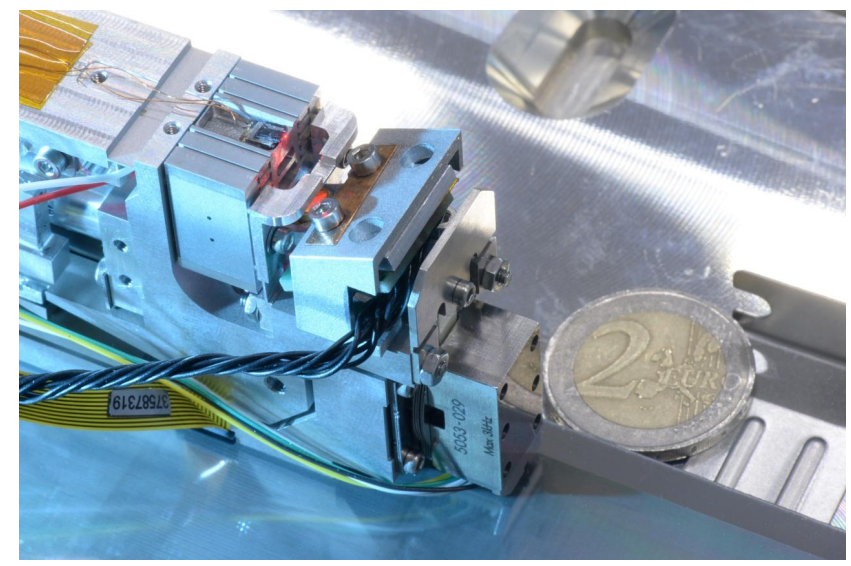

Figure 6: Picture of Miniaturized SPM demonstrator. 


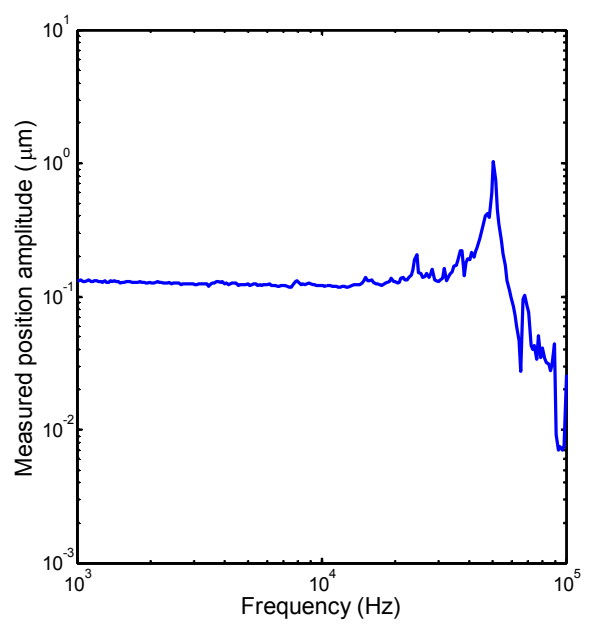

(a)

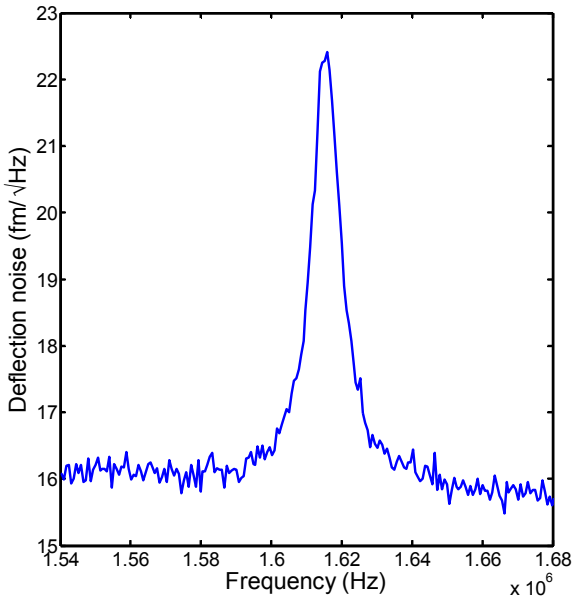

(b)

Figure 7: (a) Measured frequency spectrum of the z-scanner. (b) Measured thermal noise spectrum of the high frequency AFM probe.

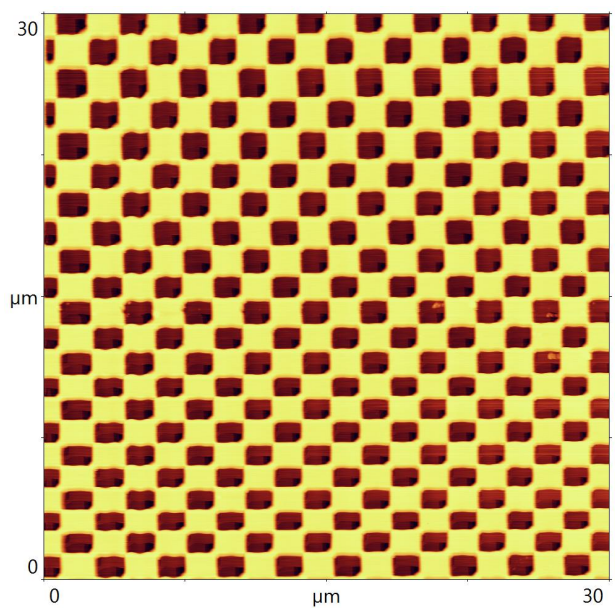

(a)

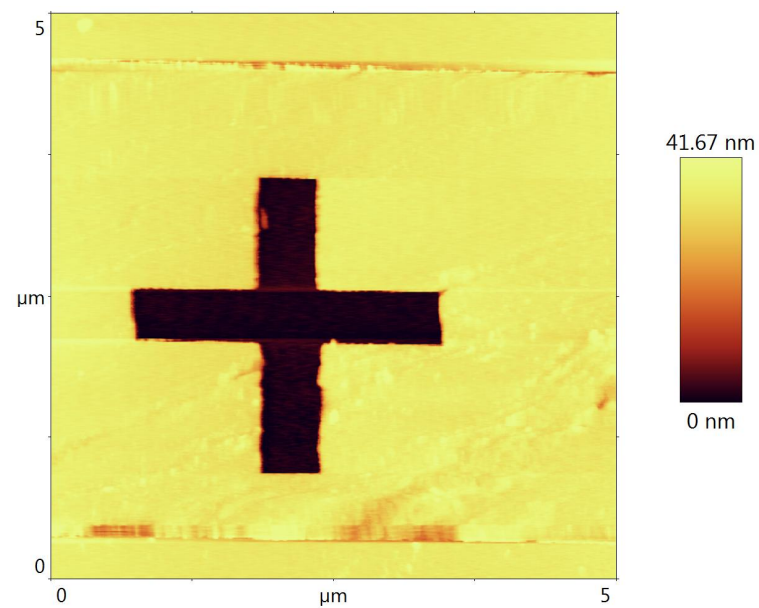

(b)

Figure 8: Images taken by MSPM. (a) TGX01 grating with $30 \mu \mathrm{m}$ pitch, $130 \mathrm{~nm}$ step. Measured using a ultra-high frequency cantilever operating at $2.12 \mathrm{MHz}, 10$ lines/s scan speed, $1024 \times 1024$ pixels. (b) Detailed view of a small alignment marker in a $\pm 50 \mathrm{~nm}$ thick resist layer.

\section{CONCLUSION AND FUTURE WORK}

In this paper we have presented the proof of principle of miniaturized scanning probe microscope and the positioning unit to be used in parallel SPM. We have shown that such a system is feasible and capable of fulfilling and exceeding the needs with respect to defect review and other inspection techniques that the semiconductor industry will have in the near future. For defect review the ITRS 2017 target of 140 sites of $10 \times 10 \mathrm{um}^{2}$ per hour will be met, and even exceeded by an order of magnitude (1500 sites/hour) if a coarse/fine scanning scheme is used. The next step to develop the demonstration of parallel SPM for a $450 \mathrm{~m}$ wafer. 


\section{REFERENCES}

[1] Jason Osborne, Shuiqing Hu, Haiming Wang, Yan Hu, , Jian Shi, Sean Hand, Chanmin Su, "High-speed atomic force microscopy for patterned defect review" in Proc. SPIE 8681, Metrology, Inspection, and Process Control for Microlithography XXVII, 86813C, San Jose, 2013.

[2] Takeshi Fukuma, Yasutaka Okazaki, Noriyuki Kodera, Takayuki Uchihashi and Toshio Ando, "High resonance frequency force microscope scanner using inertia balance support" Appl. Phys. Lett., vol. 92, p. 243119, 2008.

[3] Y Sarov, Tz Ivanov, A Frank, J-P Zöllner, N Nikolov and I W Rangelow, "Realization of cantilever arrays for parallel proximity imaging " J. Phys.: Conf. Ser, vol. 253, p. 012050, 2010.

[4] H. Sadeghian, N. B. Koster, T. C. van den Dool, "Introduction of a high throughput SPM for defect inspection and process control" Proc. SPIE, vol. 8681, p. 868127, 2013.

[5] G. Meyer, and N. M. Amer, "Novel optical approach to atomic force microscopy", Applied Physics Letters 53 (12) (1988), p. 1045-1047. 